\title{
Highly Conducting Polyacetylene Films Prepared in a Liquid Crystal Solvent
}

\author{
Kazuo AKagi, Hideki ShIRAKAWA, ${ }^{\dagger}$ Kotaro Araya, ${ }^{*}$ \\ Akio MUKOH, ${ }^{* *}$ and Toshikazu NARAHARA** \\ Institute of Materials Science, University of Tsukuba, \\ Sakura-mura, Ibaraki 305, Japan \\ *Advanced Research Laboratory, Hitachi, Ltd., \\ Kokubunji-shi, Tokyo 185, Japan \\ **Hitachi Research Laboratory, Hitachi, Ltd., \\ 4026 Kuji-cho, Hitachi-shi, Ibaraki 319-12, Japan
}

(Received November 28, 1986)

\begin{abstract}
Polyacetylene films prepared by the liquid crystal polymerization method with gravity flow technique are investigated through measurements of scanning electron micrographs, polarized infrared absorption spectra, and electrical conductivities. It is explored that the films have significant orientation of conjugated polyene chains along fibril alignment, and that upon doping by $\mathrm{I}_{2}$ or $\mathrm{AsF}_{5}$ they exhibit the highest electrical conductivity among those reported so far. By taking account of both morphological structure and activation energy for electrical conduction, the high conductivity is rationalized with preferential increase of parallel fraction of fibril achieved in the highly oriented polyacetylene films.

KEY WORDS Polyacetylene / Liquid Crystal / Scanning Electron

Micrograph / Morphology / Orientation / Polarized Infrared Spectra /

Electrical Conductivity / Anisotropy / Doping / Activation Energy /
\end{abstract}

Electrical conductivity is a central matter to be concerned in characterizing polyacetylene as low-dimensional conductive materials., ${ }^{1,2}$ Randomly oriented fibrillar morphology formed in usual as-grown polyacetylene film causes a variety of conduction pathways and screens an intrinsic conductivity of conjugated polyene chain. Since stretch-aligned polyacetylene film has been verified to exhibit an enhanced conductivity as well as an electrical anisotropy, ${ }^{3,4}$ it becomes a current focus to align fibrils in order to achieve a crucial onedimensionality for polyacetylene. Very recently, a novel method by virtue of nematic liquid crystal as polymerization solvent was developed for preparation of highly oriented polyacetylene film. ${ }^{5,6}$ Usage of the films ob- tained by this method should promise to yield deeper insights into electrical and optical properties from aspects of anisotropy. In this study, we will show that the present films used have significant orientation of polyene chains along fibril alignment, and that upon the doping $\mathrm{I}_{2}$ or $\mathrm{AsF}_{5}$ they exhibit the highest conductivity among those reported so far, primarily due to preferential increase of parallel fraction of fibril realized in highly oriented film.

\section{EXPERIMENTAL}

Polyacetylene films were prepared by using a Ziegler-Natta catalyst in a nematic liquid crystal solvent according to the procedure recently

\footnotetext{
${ }^{+}$Author to whom correspondence should be addressed.
} 
put forward. ${ }^{5,6}$ Typical concentration of the catalyst was $0.05 \mathrm{moll}^{-1}$ of $\mathrm{Ti}(\mathrm{OBu})_{4}$ and ratio of $\mathrm{AlEt}_{3}$ to $\mathrm{Ti}(\mathrm{OBu})_{4}$ was 3.0. As a liquid crystal solvent was used an equimolar mixture of 4-(trans-4-n-propylcyclohexyl)-ethoxybenzene and 4-(trans-4-n-propylcyclohexyl)butoxybenzene. The catalyst solution was aged for 30 minutes at room temperature. Six-nine grade acetylene monomer supplied from Koatsu Gas Kogyo Co., Ltd., was used without further purification. Polymerization was carried out on a vertical glass wall of a flask over which the catalyst solution flowed down by the gravity. During the polymerization, temperature was kept at $10-13^{\circ} \mathrm{C}$ in order to maintain nematic phase of the liquid crystal solution. Initial pressure of acetylene was $450-470$ Torr, and reaction time $1-120$ minutes. After polymerization, polyacetylene film was washed with toluene several times under inert gas and then immersed in an acetic acid solution (90 vol\% methanol). Thickness of polyacetylene films thus obtained was $1-30 \mu \mathrm{m}$, depending on polymerization time. Scanning electron micrograph (SEM) of the polyacetylene film was measured using a Hitachi S-540 type scanning microscope. Infrared absorption spectra of the films were measured by using a JASCO IR-810 spectrometer.

\section{RESULTS AND DISCUSSION}

As shown in Figure 1, the film exhibits a highly oriented fibrillar morphology. The orientation of fibrils was confirmed to correspond to the flow direction of the liquid crystals. Such a macroscopic alignment of fibrils is quite in contrast to the randomly oriented fibrillar morphology of usual asgrown films. ${ }^{3,6}$ This promotes us to carry out measurements of optical and electrical properties for the present films.

Convenient formulae for analysis of IR spectra were used to grasp cis and trans contents of polyacetylene films, ${ }^{1}$ i.e., $\%$ cis $=$

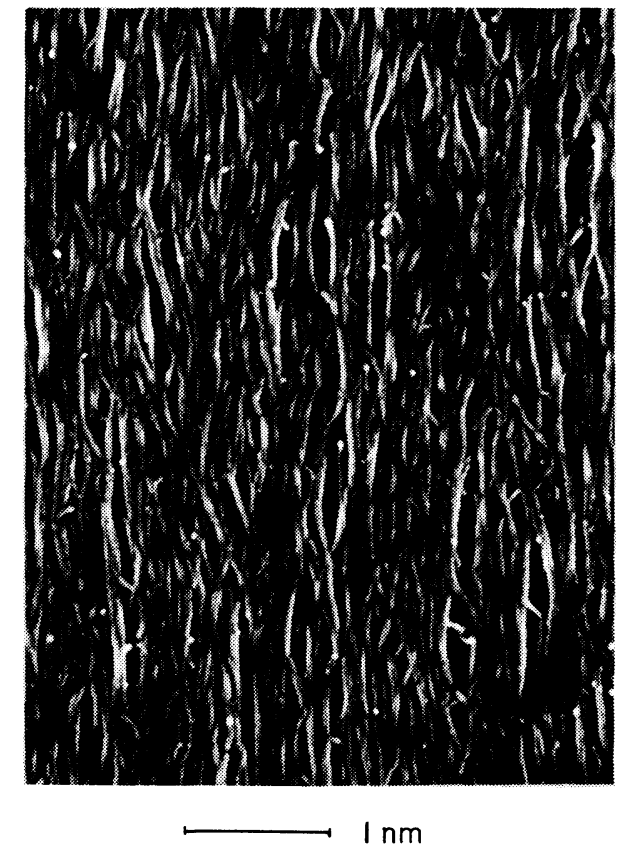

Figure 1. Scanning electron micrograph of polyacetylene film obtained by the gravity flow polymerization method.

$100 \times 1.3 A_{\text {cis }} /\left(1.3 A_{\text {cis }}+A_{\text {trans }}\right)$ and $\%$ trans $=$ $100 \times A_{\text {trans }} /\left(1.3 A_{\text {cis }}+A_{\text {trans }}\right)$, where $A_{\text {cis }}$ and $A_{\text {trans }}$ are absorbances of $c$ is and trans peaks located at 740 and $1015 \mathrm{~cm}^{-1}$, respectively. The present films are found to be composed of $53-60 \%$ cis-form and $40-47 \%$ transform. Subsequently, measurements of polarized infrared absorption spectra were carried out with polarized cells attached to the above mentioned spectrometer. Representative spectra are shown in Figure 2. Therein, $A_{\|}$and $A_{\perp}$ stand for absorbances of polarized lights parallel and perpendicular to the orientation direction of the film, respectively. It is evident that in-plane and out-ofplane vibrations of polyacetylene respectively correspond to parallel and perpendicular directions of polarized lights. ${ }^{7,8}$ It is therefore straightforward to estimate roughly an optical anisotropy with respect to each absorption peak. That is, since absorption peaks at 450 , 1330 and $3045 \mathrm{~cm}^{-1}$ are attributed to in-plane vibrations of cis-form, optical anisotropies 


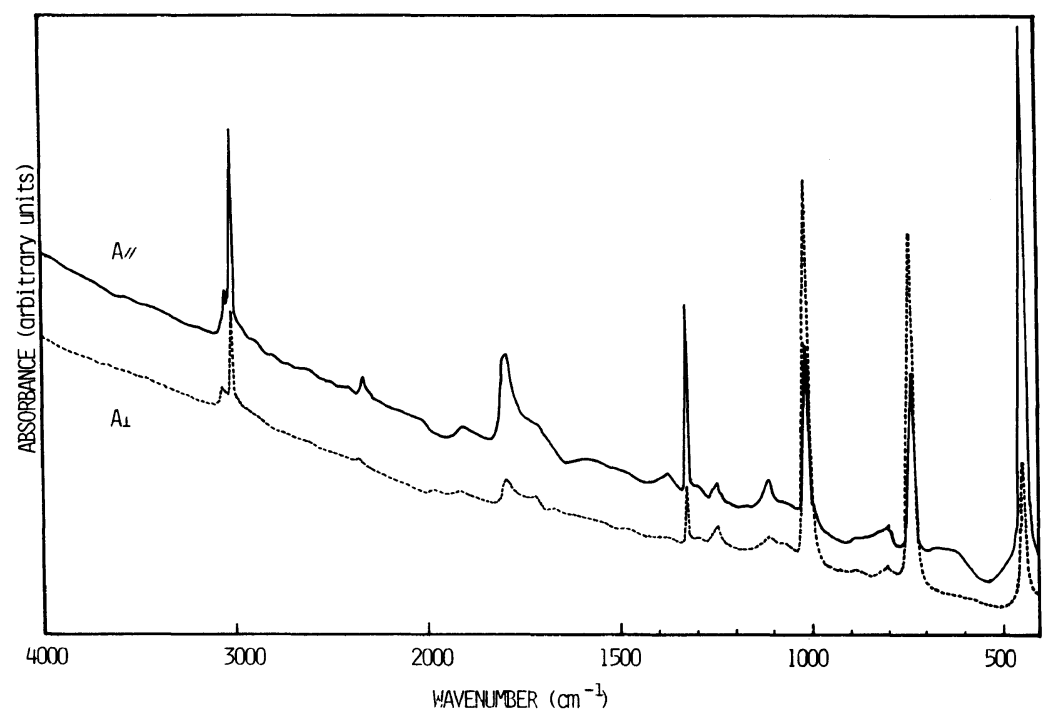

Figure 2. Polarized infrared absorption spectra of the highly oriented polyacetylene film: $A_{\|}$, parallel; $A_{\perp}$, perpendicular.

based on these peaks were calculated by $A_{\|} / A_{\perp}$. On the other hand, since those at 740 and $1015 \mathrm{~cm}^{-1}$ are respectively out-ofplane vibrations of cis- and trans-forms, anisotropies were calculated by $A_{\perp} / A_{\|}$. As a result, optical anisotropies were $2.0-3.8$, which confirms significant orientation of polyacetylene chains along fibril alignment.

The polyacetylene films were doped by exposure to vapor of iodine $\left(\mathrm{I}_{2}\right)$ or arsenic pentafluoride $\left(\mathrm{AsF}_{5}\right)$. In $\mathrm{I}_{2}$-doping vapor pressure was kept below $0.03-0.04$ Torr by cooling the dopant apparatus, while in $\mathrm{AsF}_{5}$-doping it was controlled from 0.01 to $5-7$ Torr by monitoring with digital manometer. In the latter, the doping process was stopped tentatively to evacuate such gaseous product as $\mathrm{AsF}_{3}$ which might prohibit an uniform doping. Electrical conductivity was measured with four-probe method. Figure 3 shows changes of electrical conductivities of $\mathrm{I}_{2}$-doped polyacetylene film as a function of doping time. Note that initial values of $10^{-6} \mathrm{~S} \mathrm{~cm}^{-1}$ measured before doping is located between those of $10^{-9} \mathrm{~S} \mathrm{~cm}^{-1}$ for cis-form and $10^{-5}$ $\mathrm{S} \mathrm{cm}^{-1}$ for trans-form. ${ }^{9}$ This may be due to

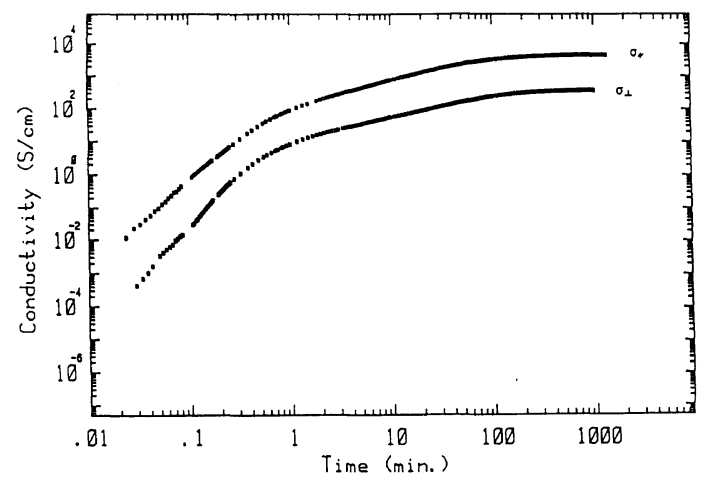

Figure 3. Electrical conductivity of the highly oriented polyacetylene film during the course of vapor phase $\mathrm{I}_{2}$ doping: $\sigma_{\|}$, parallel; $\sigma_{\perp}$, perpendicular.

the almost same contents of cis and trans forms in the present film which has been synthesized at $10-13^{\circ} \mathrm{C}$ to maintain liquid crystal phase. As the doping proceeds, both parallel and perpendicular conductivities increase with monotonous manners. Maximum values of parallel and perpendicular conductions were $4.6 \times 10^{3}$ and $3.9 \times 10^{2} \mathrm{~S} \mathrm{~cm}^{-1}$, respectively. Electrical anisotropy, defined as a ratio of parallel conductivity $\left(\sigma_{\|}\right)$to perpendicular one $\left(\sigma_{\perp}\right)$, was calculated to be 12 . These re- 
sults are summarized in Table $I$, together with those of $\mathrm{AsF}_{5}$-doping. In case of $\mathrm{AsF}_{5}$ doping, parallel and perpendicular conductivities have maximum values of $1.1 \times 10^{4}$ and $2.4 \times 10^{3} \mathrm{~S} \mathrm{~cm}^{-1}$, respectively, by which electrical anisotropy is calculated to be 5 . It must be emphasized that each maximum value of parallel conductivity attained through $\mathrm{I}_{2}$ - or $\mathrm{AsF}_{5}$-doping is the highest value among those reported so far, e.g., higher by 3 and 8 times than those of stretched aligned films and randomly oriented films, respectively. ${ }^{10}$ Especially, the conductivity with order of $10^{4}$ $\mathrm{Scm}^{-1}$ attained by the $\mathrm{AsF}_{5}$-doping corresponds to that of Mercury $\left(1.04 \times 10^{4} \mathrm{~S} \mathrm{~cm}^{-1}\right.$ at $0^{\circ} \mathrm{C}$ ) and is also close to an estimated value

Table I. Electrical conductivity and anisotropy of highly oriented polyacetylene film doped with $\mathrm{I}_{2}$ and $\mathrm{AsF}_{5}$

\begin{tabular}{|c|c|c|c|}
\hline \multirow{2}{*}{$\begin{array}{l}\text { Dopant } \\
\text { species }\end{array}$} & $\sigma_{\|}$ & $\sigma_{\perp}$ & \multirow{2}{*}{$\sigma_{\|} / \sigma_{\perp}$} \\
\hline & $\mathrm{S} \mathrm{cm}^{-1}$ & $\mathrm{~S} \mathrm{~cm}^{-1}$ & \\
\hline $\mathrm{I}_{2}$ & $4.6 \times 10^{3}$ & $3.9 \times 10^{2}$ & 12 \\
\hline $\mathrm{AsF}_{5}$ & $1.1 \times 10^{4}$ & $2.4 \times 10^{3}$ & 5 \\
\hline
\end{tabular}

of intrinsic conductivity in heavily $\mathrm{AsF}_{5}$ doped polyacetylene with metallic state ( $c a$. $4 \times 10^{4} \mathrm{~S} \mathrm{~cm}^{-1}$ at room temperature).$^{10}$

It is of interest to note that the electrical anisotropy of 12 obtained for the present $\mathrm{I}_{2}$ doped film is comparable to those $(10-13)$ of $\mathrm{I}_{2}$-doped stretched films with elongation ratio of 2.9.,4 However, the conductivity of the forms is 3 times higher than that of the latter, as mentioned above. These results may indicate that the present film has apparently the same degree of fibril alignment as in the case of mechanically stretched films by three times, but that it is free from cleavage of fibrils encountered in mechanical elongation of film and also has less interfibril contacts to cause various conduction pathways and barriers for conduction.

Figure 4 shows temperature dependence of normalized conductivity in the $\mathrm{AsF}_{5}$-doped polyacetylene film in the range of room temperature down to liquid nitrogen temperature. Both parallel and perpendicular conductivities are found to decrease gradually with decreasing temperature. Arrhenius plots gave approximately straight lines in the range of 250

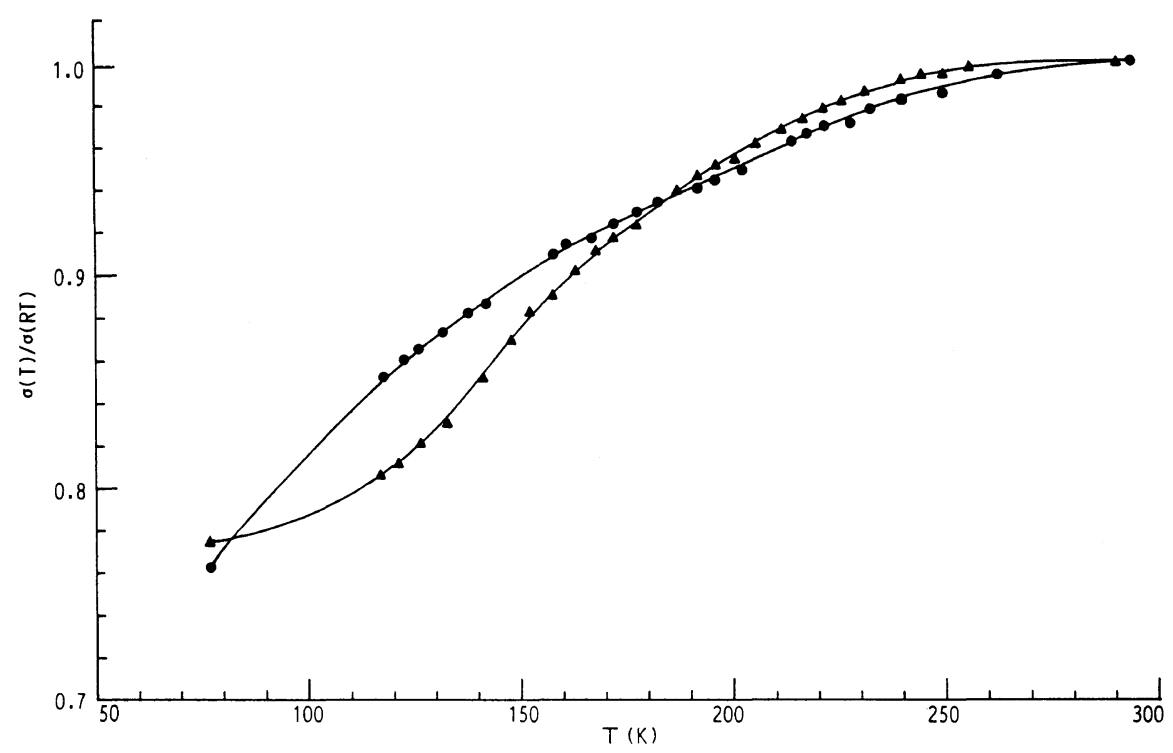

Figure 4. Temperature dependence of normalized conductivity of $\mathrm{AsF}_{5}$-doped highly oriented polyacetylene film: $\boldsymbol{O}$, parallel; $\boldsymbol{\Delta}$, perpendicular. 
$125 \mathrm{~K}$. Activation energies calculated for parallel and perpendicular conductions were 3.9 and $4.3 \mathrm{meV}$, respectively. Such small activation energies unambiguously indicate a socalled 'metallic state' in doped polyacetylene films. ${ }^{10}$ In spite of significant anisotropy of $c a$. 5 in electrical conductivity, there is only small difference in activation energies between parallel and perpendicular directions. This implies a similar role of interfibril contacts in each direction, in agreement with the case of stretch-aligned films. ${ }^{10,11}$ It is therefore argued that the high electrical conductivities achieved in the present film is primarily due to high orientation of fibrils, allowing a preferential increase of fibril fraction in the parallel direction and decrease in the perpendicular one.

In conclusion, we have first attained the electrical conductivity of $10^{4} \mathrm{~S} \mathrm{~cm}^{-1}$ by using $\mathrm{AsF}_{5}$-doped highly oriented polyacetylene film prepared through liquid crystal polymerization method. These results demonstrate profound potentialities of highly oriented polyacetylene films as organic conductors, leading us to an advanced stage of onedimensional conductive polymers.

Acknowledgments. The authors wish to acknowledge the technical support of $\mathrm{Mr}$. H.
Sakai. Thanks are also due to Koatsu Gas Kogyo Co., Ltd., for supplying the highly purified acetylene gas. Work at University of Tsukuba was supported by Grant-in-Aids of special research projects on organic thin films for information conversion (No. 60111003, 61103003) and in part on properties of molecular assembles (No. 60104001).

\section{REFERENCES}

1. T. Ito, H. Shirakawa, and S. Ikeda, J. Polym. Sci., Polym. Chem. Ed., 12, 11 (1974).

2. J. C. W. Chien, "Polyacetylene Chemistry, Physics, and Material Science," Academic Press, New York, N. Y., 1984.

3. H. Shirakawa and S. Ikeda, Synth. Met., 1, 175 (1979/80).

4. Y.-W. Park, M. A. Druy, C. K. Chiang, A. G. MacDiarmid, A. J. Heeger, H. Shirakawa, and S. Ikeda, J. Polym. Sci., Polym. Lett. Ed., 17, 195 (1979).

5. K. Araya, A. Mukoh, T. Narahara, and $H$. Shirakawa, Chem. Lett., 1141 (1984).

6. K. Araya, A. Mukoh, T. Narahara, and H. Shirakawa, Synth. Met., 14, 199 (1986).

7. H. Shirakawa and S. Ikeda, Polym. J., 2, 231 (1971).

8. M. Galtier, M. Charbonnel, A. Montaner, and J. L. Ribet, Polymer, 12, 1253 (1984).

9. H. Shirakawa, T. Ito, and S. Ikeda, Makromol. Chem., 179, 1565 (1978).

10. Y.-W. Park, A. J. Heeger, M. A. Druy, and A. G. MacDiarmid, J. Chem. Phys., 73, 946 (1980).

11. Y.-C. Chen, K. Akagi, and H. Shirakawa, Synth. Met., 14, 173 (1986). 